\title{
Evidências de validade e fidedignidade da Escala Internacional de Inteligência Leiter-R para crianças dos 6 aos 8 anos
}

\author{
Tatiana Pontrelli Mecca ${ }^{1}$ \\ Centro Universitário FIEO \\ Lucas de Francisco Carvalho \\ Universidade São Francisco. \\ Mário Rodrigues Simões \\ Universidade de Coimbra \\ Elizeu Coutinho Macedo \\ Universidade Presbiteriana Mackenzie
}

\begin{abstract}
RESUMO - Este estudo verificou os parâmetros psicométricos da escala não-verbal de inteligência Leiter-R. Esta foi administrada em 213 crianças com idade entre 6 e 8 anos. Análise fatorial exploratória e índices de ajustes confirmatórios mostraram adequação da estrutura interna para dois fatores conforme a versão original do instrumento. Foi verificada estabilidade temporal, com correlações de magnitude moderada a alta entre teste e re-teste. Coeficientes de Kuder-Richardson e SpearmanBrown variaram entre os subtestes em função das diferentes idades. Maiores valores foram observados nos subtestes Sequências e Padrões Repetidos para todas as idades. Aumento significativo dos escores ocorreu apenas dos 6 para os 7 e 8 anos. Foram verificadas evidências de validade convergente com a WISC-III e as Matrizes Progressivas Coloridas de Raven.
\end{abstract}

Palavras-Chave: inteligência, psicometria, Leiter-R, infância

\section{Validity and Reliability of the Leiter International Performance Scale - Revised for children of 6 to 8 years}

\begin{abstract}
This study verified psychometric parameters of the Leiter-R, a nonverbal intelligence scale. The scale was administered to 213 children between 6 and 8 years. Exploratory factor analysis and confirmatory indexes showed adequacy for a two factor structure according to the original version. Moderate and high correlations between test-retest measures indicated the stability of the test scores. The values of Kuder-Richardson and Spearman-Brown coefficients varied between subtests according to the age of the children. In all age groups the highest values were observed for the subtests Sequential Order and Repeated Patterns. Significant increase in scores occurred only from 6 to 7 and 8 years. Evidence of convergent validity was obtained between the Leiter-R with the WISC-III and the Raven Colored Progressive Matrices.
\end{abstract}

Keywords: intelligence, psychometrics, Leiter-R, childhood

Os testes tradicionais que avaliam inteligência exigem habilidades que determinados grupos não desenvolveram de forma adequada e, consequentemente, sua aplicação se torna inviável ou muito limitada (Mecca, 2010; Roid \& Miller, 1997). Uma das limitações existentes se deve ao fato de que os instrumentos atualmente disponíveis possuem características em sua padronização que dificultam a avaliação de determinados grupos clínicos (Antonio, Mecca \& Macedo, 2012), como, por exemplo, o uso de instruções verbais e respostas que requerem habilidades motoras finas ou de linguagem. Uma das alternativas é a Escala Internacional de Inteligência Leiter-R (Leiter-R).

A utilização da Leiter-R está comumente associada aos estudos de avaliação cognitiva de indivíduos com transtornos do desenvolvimento e que apresentam dificuldades em responder aos testes tradicionais. As instruções e formas de responder não requerem compreensão e expressão verbais, sendo uma vantagem na avaliação de algumas condições, tais como nos Transtornos do Espectro do Autismo, Distúrbios de

1 Endereço para correspondência: Rua Piauí, n.181, $10^{\circ}$ andar, Laboratório de Neurociência Cognitiva e Social, Consolação, São Paulo, SP, Brasil. CEP: 01302-000.E-mail: tati.mecca@gmail.com
Linguagem, Deficiência Auditiva, entre outras (Antonio et al., 2012). Além disso, os estímulos são universais, diminuindo as especificidades de diferentes contextos culturais (Anastasi \& Urbina, 2000).

A Leiter-R é um instrumento composto por duas baterias, sendo uma de inteligência (Bateria de Visualização Raciocínio - BVR) e a outra de memória e atenção (Bateria $\mathrm{AM}$ ), além de mais quatro escalas comportamentais que definem um perfil do indivíduo. $O$ teste pode ser utilizado em uma faixa etária abrangente, dos 2 aos 20 anos. A primeira parte é composta pela BVR, cujo resultado se traduz em uma medida global do nível intelectual e é objeto de investigação do presente estudo. É composta por dez subtestes: FiguraFundo, Formas Completas, Sequências, Padrões Repetidos, Pareamento, Analogias, Contexto da Figura, Classificação, Dobra de Papel e Rotação de Figuras. No entanto, apenas os quatro primeiros são comuns a todas as faixas etárias, compondo o Quociente Intelectual (QI) Estimado. Enquanto que o QI Total é composto pelas quatro provas referidas e mais duas, sendo estas específicas a cada grupo etário. Dos 2 aos 5 anos, aplicam-se Pareamento e Classificação; dos 6 aos 20, são administrados Analogias e Dobra de Papel; a partir dos 11 anos, aplica-se o subteste Rotação de Figuras 
(Roid \& Miller, 1997). Em função dos objetivos deste estudo, serão enfatizados os subtestes destinados a crianças na faixa etária dos 6 aos 8 anos.

O subteste Figura-Fundo avalia atenção e discriminação visual, uma vez que o objetivo é encontrar a figura-alvo em uma prancha composta por diversos estímulos que podem ser semelhantes e, por isso, distratores ao estímulo-alvo. Já o subteste Formas Completas avalia a capacidade de síntese visual e a tarefa consiste em identificar o estímulo-alvo, que é apresentado em desenhos fragmentados, e encontrá-lo em uma prancha. Em Sequências, o indivíduo deve selecionar o estímulo que completa uma sequência corretamente, seguindo uma progressão lógica, avaliando-se o raciocínio sequencial. No subteste Padrões Repetidos, em que o indivíduo deve descobrir a regra subjacente à sequência de estímulos e completá-la selecionando os estímulos corretos, avalia-se o raciocínio indutivo. No subteste Analogias, o indivíduo deve selecionar qual o estímulo que completa corretamente uma matriz, avaliando-se também o raciocínio indutivo e a habilidade visuo-espacial nos itens que demandam rotação mental. Por fim, o subteste Dobra de Papel avalia a capacidade visuo-espacial a partir de rotação de figuras. $\mathrm{O}$ objetivo neste subteste é que o indivíduo encontre, em uma prancha com diversos estímulos, aquele que representaria o estímulo-alvo caso ele estivesse dobrado.

Esse instrumento foi construído a partir do modelo psicométrico proposto por John Carroll, denominado Teoria dos Três Estratos, segundo o qual as habilidades cognitivas estão dispostas em uma estrutura hierárquica (Carroll, 1993). Na primeira camada, estão habilidades muito específicas, tais como aquelas avaliadas nos itens dos testes (e.g., raciocínio indutivo e sequencial). Na segunda camada, estão habilidades amplas que agrupam as habilidades específicas, como a Inteligência Fluida, que contempla o raciocínio indutivo, sequencial e quantitativo, por exemplo. Já na terceira e última camada, está o fator geral de inteligência, denominado incialmente por Spearman de fator $\mathrm{g}$.

A Leiter-R avalia especificamente a inteligência fluida $(G f)$ e o processamento visual $(G v)$, que são habilidades amplas da Camada II do modelo de Carroll (Roid \& Miller, 1997). A Gf se refere a habilidades de raciocínio em situações novas, minimamente dependentes de conhecimentos adquiridos, englobando também a resolução de problemas, a correlação de ideias, a indução de conceitos abstratos e a compreensão de implicações. Exemplos de fatores específicos da Camada I relacionados ao fator amplo Gf são os raciocínios sequenciais, quantitativos e indutivos. Já o fator amplo Gv se refere a gerar, reter e manipular imagens visuais abstratas e seus fatores específicos da Camada I, que estão relacionados a tarefas de visualização e relações espaciais (Carroll, 1993).

Em função do grupo etário objeto de investigação do presente estudo, serão descritos, a seguir, os parâmetros psicométricos da Leiter-R para crianças entre 6 e 8 anos de idade. De acordo com o modelo teórico (Carroll, 1993), os subtestes podem ser agrupados em dois grandes fatores: Gf e Gv. Os subtestes que formam o fator Gf avaliam habilidades específicas como raciocínio sequencial (Sequências) e raciocínio indutivo (Padrões Repetidos). O fator $\mathrm{Gv}$ é formado por quatro tarefas específicas que avaliam discriminação e exploração visual (Figura-Fundo), habilidade de fechamento e síntese (Formas Completas), bem como habilidades visuoespaciais (Analogias e Dobra de Papel).

Os resultados observados a partir de análise fatorial exploratória (AFE) com a amostra americana revelaram a presença de dois fatores. O primeiro (Gv) é composto por maiores cargas nos subtestes Figura-Fundo e Analogias, enquanto o segundo (Gf) é composto pelos subtestes Formas Completas, Sequências e Padrões Repetidos. O subteste Dobra de Papel apresentou cargas baixas em ambos os fatores, embora a carga mais alta tenha sido para o segundo fator (Roid \& Miller, 1997). Esses achados apontam que os dados empíricos diferem parcialmente do modelo proposto, pois o subteste Formas Completas apresentou maior carga no fator Gf, juntamente com Sequências e Padrões Repetidos. O subteste Dobra de Papel apresentou cargas muito baixas em ambos os fatores, embora a maior carga também esteja associada ao fator Gf (Roid \& Miller, 1997), diferentemente do modelo teórico apresentado.

Em estudo prévio com crianças aos 6 anos de idade, pré-escolares, AFE revelou a presença de dois fatores que juntos explicaram 62,19\% da variância na Leiter-R (Mecca, 2010). O subteste Sequências apresentou maior carga no primeiro fator, juntamente com os subtestes Figura-Fundo e Analogias. Já o segundo fator foi composto pelos subtestes Formas Completas e Padrões Repetidos. De modo geral, os dados são semelhantes à amostra americana, com exceção do subteste Sequências. Semelhante ao verificado por Roid e Miller (1997), o estudo de Mecca (2010) apontou para menores cargas fatoriais do subteste Dobra de Papel em ambos os fatores, embora a carga maior tenha sido verificada no segundo fator juntamente com os subtestes Formas Completas e Padrões Repetidos. Apesar de satisfeitos os critérios para a análise fatorial, no estudo de Mecca, os resultados se referem a um número reduzido de sujeitos e o aumento da amostra e das faixas etárias são necessários para consistência dos achados. Além disso, as análises realizadas com a população americana se referem às idades dos 6 aos 10 anos, enquanto que os dados de Mecca se referem a crianças com 6 anos de idade.

Evidências de validade convergente da Leiter-R com a Escala de Inteligência Stanford-Binet indicaram correlações de magnitude moderada a alta entre alguns subtestes, especificamente com relação ao subteste da StanfordBinet que avalia habilidades de processamento visual e inteligência fluida (Gf) (Anastasi, 1977; Roid \& Miller, 1997). Cathers-Schiffmane e Thompson (2007) verificaram evidências de validade convergente da Leiter-R com a Escala de Inteligência Wechsler para Crianças (WISC-III) em dois grupos de crianças com idades entre 8 e 13 anos, sendo que o primeiro tinha o inglês como língua materna e o segundo, o espanhol. Os resultados indicaram que, nos dois grupos, a correlação entre QI Total na Leiter-R e QI Total na WISC-III foi positiva, significativa e de magnitude moderada. Também foram verificadas correlações positivas e significativas entre o QI Total na Leiter-R com os índices fatoriais de Organização Perceptual e Compreensão Verbal da WISC-III, em ambos os grupos. O mesmo padrão de resultados, com valores semelhantes, foram observados em correlações parciais controlando o nível socioeconômico. 
No manual técnico da Leiter-R, os autores (Roid \& Miller, 1997) apresentam dois estudos de evidências de validade com a WISC-III. No primeiro, há correlações elevadas com o QI Total, com o QI de Execução e com o QI Verbal da WISCIII. No segundo, foram observados valores semelhantes, com correlações elevadas entre o QI Total na Leiter-R e o QI Total e QI de Execução da WISC-III. No entanto, a correlação com o QI Verbal foi de magnitude moderada .

Hooper e Bell (2006), ao investigarem possíveis relações entre a Leiter-R e o Universal Nonverbal Intelligence Test, observaram uma correlação moderada, positiva e significativa entre os escores desses instrumentos. O estudo de Mecca, Antônio, Seabra e Macedo (2014) também encontrou correlações positivas, estatisticamente significativas e de magnitude moderada entre os escores brutos obtidos na Leiter-R e nas Matrizes Progressivas Coloridas de Raven, bem como na Escala de Maturidade Mental Colúmbia para crianças pré-escolares. Os estudos mostraram correlações moderadas entre a Leiter-R e testes não-verbais, indicando que os desempenhos nesses instrumentos estão, em parte, relacionados a uma habilidade comum, enquanto outra parte pode ser explicada por habilidades específicas relacionadas à demanda de cada teste.

Em relação à fidedignidade do instrumento, Roid e Miller (1997) apresentam análises de consistência interna por faixa etária e medidas de estabilidade para cada subteste. Os valores de Kuder-Richardson aos 6 anos variam entre 0,73 para Dobra de Papel e 0,88 para Formas Completas. Aos 7 e aos 8 anos, o menor valor também foi para Dobra de Papel $(0,67)$ e o maior para Formas Completas $(0,87$ e 0,84 , respectivamente). Aos 8 anos, juntamente com Formas Completas, o subteste Analogias também apresenta consistência de 0,84 . As correlações entre teste e re-teste foram positivas, de magnitude moderada a alta, variando entre 0,70 para o subteste Dobra de Papel e 0,83 para o subteste Formas Completas. De modo geral, esses dados revelam maior precisão para o subteste Formas Completas e menor para o subteste Dobra de Papel, embora este ainda esteja dentro dos parâmetros psicométricos adequados conforme Urbina (2014) e os Standards for Educational and Psychological Testing (American Educational Research Association, American Psychological Association, \& National Council on Measurement in Education, 2014).

Diferentemente da estabilidade verificada por Roid e Miller (1997), os resultados do estudo de Mecca et al. (2014), com 45 crianças pré-escolares entre 4 e 6 anos de idade, apresentaram valores menores de correlação entre teste e re-teste. Correlações significativas e de magnitude moderada foram observadas na maioria dos subtestes, com exceção de Sequências, em que não houve correlação significativa entre os desempenhos na primeira e na segunda aplicação.

Deve-se considerar que não foram encontradas publicações, em âmbito nacional, evidenciando as propriedades psicométricas da Bateria de Raciocínio e Visualização da Leiter-R em crianças de 6 a 8 anos de idade. Sendo assim, o objetivo do presente estudo foi buscar evidências de validade e fidedignidade da versão brasileira da Leiter-R em crianças dessa faixa etária.

\section{Método}

\section{Participantes}

Foram avaliadas 213 crianças de Ensino Infantil e Fundamental I, entre 6 e 8 anos de idade $(M=6,89$; $\mathrm{DP}=0,82)$. Os grupos etários foram compostos por 85 crianças com 6 anos (39,90\%), 66 com 7 anos (31\%) e 62 com 8 anos (29,10\%). Participaram do estudo 120 meninos $(56,30 \%)$ e 93 meninas $(43,70 \%)$. A amostra foi selecionada por conveniência, de acordo com a disponibilidade e a autorização das instituições e responsáveis pelas crianças. Quatro escolas localizavam-se na Zona Norte; uma, na Zona Leste; e uma, na Zona Oeste da cidade de São Paulo. Foram excluídas crianças com deficiências sensoriais conhecidas e não corrigidas de acordo com as informações obtidas nas próprias escolas.

\section{Instrumentos}

Foram aplicados os seis subtestes da BVR da Leiter-R que compõem o QI Total para crianças na faixa etária dos 6 aos 8 anos de idade, a saber: Figura-Fundo, Analogias, Formas Completas, Sequências, Padrões Repetidos e Dobra de Papel. A Tabela 1 apresenta a caracterização dos subtestes.

A aplicação da Leiter-R é individualizada, com duração em torno de 30 minutos para cada criança. As respostas aos itens de todos os subtestes são dicotômicas, ou seja, 0 (zero) para erro e 1 para acerto. Todos os subtestes possuem itens de treino nos quais a instrução pode ser dada até três vezes para garantir que a criança entenda a demanda. Os itens não possuem tempo limite de aplicação. O tempo é marcado em apenas alguns itens dos subtestes Analogias e Dobra de Papel, para que a criança possa ganhar pontos bônus se conseguir realizar o item em um tempo determinado. As interrupções de cada subteste são feitas após erros que são acumulados e não necessariamente consecutivos. Para a aplicação, a criança senta-se de frente para o examinador, o qual coloca sobre a mesa a prancha de estímulos (na vertical). Os cartões de cada item são apresentados entre a prancha e a criança.

Para além da Leiter-R, foram aplicadas as Matrizes Progressivas Coloridas de Raven (Angelini, Alves, Custódio, Duarte, \& Duarte, 1999) e a WISC-III (Figueiredo, 2002). Ambas fornecem uma medida de inteligência para crianças.

\section{Procedimentos}

A coleta de dados ocorreu nas próprias escolas, após assinatura do Termo de Consentimento Livre e Esclarecido pelos responsáveis, durante o período de aula, conforme combinado previamente com a instituição, de forma a não prejudicar as atividades escolares dos alunos. Todos os instrumentos foram aplicados individualmente, em dois encontros com cada criança. No primeiro encontro, foi realizada a aplicação da Leiter-R (com duração de 30 minutos) e, no segundo encontro, foi aplicada a WISC-III em crianças no Ensino Fundamental (duração de 60 minutos) ou 
as Matrizes Progressivas Coloridas de Raven (duração de 10 a 12 minutos) em crianças do Ensino Infantil.

As análises foram realizadas considerando os escores brutos da Leiter-R. Para verificação de evidências de validade com base na estrutura interna, procedeu-se à AFP para variáveis policóricas por meio do software MPlus versão 6.12. O número de fatores a serem mantidos na análise foi verificado com base na análise paralela (Watkins, 2006). Para veiculação da análise paralela a partir de variáveis policóricas, foi utilizado o software R versão 2.15.3.

Para avaliar a fidedignidade do instrumento, foi calculada a estabilidade a partir da correlação teste e re-teste, com um mês de intervalo entre as duas aplicações. Também foi verificada a homogeneidade dos itens a partir do Método das Metades, com correção pela fórmula de Spearman-Brown. As metades foram divididas em itens ímpares e pares de modo que, em ambas as metades, fossem incluídos itens fáceis e difíceis. Por fim, para verificação da consistência interna, foi realizado o cálculo do coeficiente de Kuder-Richardson de cada subteste de acordo com as faixas etárias.
Evidências de validade utilizando a idade como critério foram verificadas a parir de análises de variância univariadas (ANOVA), comparando o desempenho entre os grupos etários. Também foram investigadas evidências de validade convergente a partir de correlações parciais, controlando o efeito da idade, entre a Leiter-R e a WISC-III, sendo utilizadas as medidas gerais, bem como subtestes específicos. O mesmo foi realizado com as Matrizes Progressivas Coloridas de Raven, sendo considerados os escores brutos das séries $\mathrm{A}, \mathrm{Ab}, \mathrm{B}$ e a pontuação bruta total.

Para análise dos resultados relacionados à fidedignidade e das evidências de validade baseadas na relação com critérios externos, foi utilizado o programa Statistical Package for Social Sciences ®versão 18.0 para Windows (SPSS Inc).

\section{Resultados}

A fim de analisar a estrutura interna da Leiter-R, foi realizada AFE. A análise paralela sugeriu a extração de dois fatores, seguindo-se AFE que resultou nas cargas fatoriais

Tabela 1. Descrição dos subtestes da Leiter-R e habilidades avaliadas

\begin{tabular}{|c|c|c|c|c|c|c|}
\hline Subteste & \multicolumn{3}{|c|}{ Tarefa } & \multicolumn{3}{|c|}{ O Que Avalia } \\
\hline Figura-Fundo & \multicolumn{3}{|c|}{$\begin{array}{l}\text { Encontrar nas pranchas as figuras que correspondem } \\
\text { aos cartões }\end{array}$} & \multicolumn{3}{|c|}{ Discriminação, reconhecimento e retenção visual. } \\
\hline Analogias & \multicolumn{3}{|c|}{ Completar uma matriz com um ou mais cartões. } & \multicolumn{3}{|c|}{ Raciocínio indutivo e habilidade visuo-espacial } \\
\hline Formas Completas & \multicolumn{3}{|c|}{$\begin{array}{l}\text { Reconhecer um objeto inteiro na prancha através da } \\
\text { apresentação de partes fragmentadas nos cartões. }\end{array}$} & \multicolumn{3}{|c|}{ Percepção, capacidade de síntese, rotação mental. } \\
\hline Sequências & \multicolumn{3}{|c|}{$\begin{array}{l}\text { Completar as figuras na prancha selecionando os } \\
\text { estímulos que progridem numa determinada ordem. }\end{array}$} & \multicolumn{3}{|c|}{$\begin{array}{l}\text { Raciocínio indutivo para a capacidade de fazer } \\
\text { progressões lógicas através de figuras }\end{array}$} \\
\hline Padrões Repetidos & \multicolumn{3}{|c|}{$\begin{array}{l}\text { Identificar padrões repetitivos através de figuras e } \\
\text { completá-los seguindo a sequência apresentada na } \\
\text { prancha. }\end{array}$} & \multicolumn{3}{|c|}{ Raciocínio indutivo. } \\
\hline Dobrar Papel & \multicolumn{3}{|c|}{$\begin{array}{l}\text { Dobrar ou desdobrar mentalmente um objeto } \\
\text { bidimensional apresentado no cartão e fazer } \\
\text { corresponder a um alvo na prancha. }\end{array}$} & \multicolumn{3}{|c|}{$\begin{array}{l}\text { Habilidades visuo-espaciaise raciocínio indutivo e } \\
\text { dedutivo. }\end{array}$} \\
\hline \multicolumn{7}{|c|}{ Tabela 2. Sumário do modelo e das análises fatoriais exploratórias } \\
\hline \multirow{2}{*}{ Subtestes } & \multicolumn{2}{|c|}{ Modelo teórico proposto } & \multicolumn{2}{|c|}{ Modelo empírico americano } & \multicolumn{2}{|c|}{ Modelo empírico exploratório } \\
\hline & Fator 1 & Fator 2 & Fator 1 & Fator 2 & Fator 1 & Fator 2 \\
\hline Figura-Fundo & & & 0,47 & & 0,56 & \\
\hline Formas Completas & & & & 0,52 & 0,85 & \\
\hline Sequências & & & & 0,42 & & 0,76 \\
\hline Padrões Repetidos & & & & 0,60 & 0,50 & \\
\hline Analogias & & & 0,33 & & & 0,60 \\
\hline Dobra de Papel & & & & 0,12 & & 0,55 \\
\hline \multicolumn{7}{|l|}{ Dados confirmatórios } \\
\hline$X 2 / d f$ & & & \multicolumn{2}{|c|}{2,01} & \multicolumn{2}{|c|}{0,50} \\
\hline RMSEA & & & \multicolumn{2}{|c|}{0,06} & \multicolumn{2}{|c|}{0,00} \\
\hline$C F I$ & & & \multicolumn{2}{|c|}{0,98} & \multicolumn{2}{|c|}{1,00} \\
\hline$S M R$ & & & \multicolumn{2}{|c|}{0,02} & \multicolumn{2}{|c|}{0,01} \\
\hline$B I C$ & & & \multicolumn{2}{|c|}{6989,417} & \multicolumn{2}{|c|}{7056,960} \\
\hline$\alpha$ & & & \multicolumn{2}{|c|}{$0,560,73$} & \multicolumn{2}{|c|}{$0,770,55$} \\
\hline
\end{tabular}


descritas na Tabela 2. Esta também apresenta os índices de ajuste relacionados aos dados confirmatórios.

De acordo com os dados apresentados na Tabela 2, os resultados obtidos a partir da AFE indicam que o modelo empírico da amostra do presente estudo é composto por maiores cargas fatoriais dos subtestes Figura-Fundo, Formas Completas e Padrões Repetidos em um fator, enquanto que, no segundo, verificaram-se maiores cargas nos subtestes Sequências, Analogias e Dobra de Papel. Entretanto, observaram-se algumas diferenças em relação modelo teórico proposto na Leiter-R, em que os subtestes Analogias e Dobra de Papel se agrupam no mesmo fator (Gv) que Figura-Fundo e Formas Completas, enquanto que Sequências e Padrões Repetidos seriam parte do segundo fator (Gf).

Também há diferenças em relação ao modelo empírico obtido a partir dos dados da amostra normativa americana, em que os subtestes Formas Completas e Padrões Repetidos apresentam maior carga fatorial no fator Gf juntamente com Sequências e Dobra de Papel. Nesse mesmo modelo, o subteste Analogias apresenta maior carga no fator Gv junto com Figura-Fundo. Apesar das diferenças observadas, os dados confirmatórios revelaram melhor adequação dos índices de ajuste em relação ao modelo empírico observado quando comparados aos modelos teórico e empírico da amostra americana.

No que tange à fidedignidade, como medida de estabilidade, buscou-se o coeficiente de Correlação de Pearson entre duas aplicações da Leiter-R. As médias e os desvios-padrão de ambas as aplicações bem como os dados obtidos a partir das análises de correlação encontram-se sumariados na Tabela 3.

Tabela 3. Estatísticas descritivas, Correlação de Pearson e significância entre as medidas de teste e re-teste

\begin{tabular}{lccc}
\hline \multicolumn{1}{c}{ Subtestes } & $\begin{array}{c}\text { Média da 1 }^{\mathbf{a}} \\
\text { aplicação }(\boldsymbol{D P})\end{array}$ & $\begin{array}{c}\text { Média da 2 }^{\mathbf{a}} \\
\text { aplicação }(\boldsymbol{D P})\end{array}$ & $\begin{array}{c}\text { Correlação } \\
(\boldsymbol{r})\end{array}$ \\
\hline Figura-Fundo & $17,14( \pm 4,88)$ & $16,91( \pm 3,85)$ & $0,80^{*}$ \\
Analogias & $5,14( \pm 2,96)$ & $5,77( \pm 3,85)$ & $0,70^{*}$ \\
Formas Completas & $23,41( \pm 5,17)$ & $24,86( \pm 4,87)$ & $0,51^{*}$ \\
Sequências & $13,14( \pm 7,25)$ & $14,55( \pm 9,42)$ & $0,64^{*}$ \\
Padrões Repetidos & $12,53( \pm 5,50)$ & $13,18( \pm 5,63)$ & $0,74^{*}$ \\
Dobra de Papel & $4,27( \pm 1,66)$ & $5,14( \pm 1,80)$ & $0,68^{*}$ \\
Total & $75,82( \pm 21,40)$ & $79,91( \pm 23,91)$ & $0,81^{*}$ \\
\hline
\end{tabular}

$*_{p} \leq 0,01$
Observa-se que, de modo geral, as médias obtidas na segunda aplicação foram discretamente maiores em relação à primeira aplicação da Leiter-R. Entretanto, os resultados obtidos a partir das análises de Correlação de Pearson indicaram relações positivas e de magnitude moderada nos subtestes Analogias, Formas Completas, Sequências, Padrões Repetidos e Dobra de Papel. Já entre os escores totais e no subteste Figura-Fundo, as correlações foram de alta magnitude. As correlações foram estatisticamente significativas em todas as medidas.

A homogeneidade dos itens foi verificada pelo Método das Metades. Os coeficientes de Spearman-Brown de cada subteste em função da faixa etária e da amostra total estão descritos na Tabela 4. Entre parênteses, são apresentadas as correlações entre as duas metades.

Os valores obtidos a partir do Coeficiente de SpearmanBrown, sendo a maior parte acima de 0,80 , indicam que os subtestes são fidedignos em relação à homogeneidade dos itens. Apenas o subteste Analogias para crianças com 6 anos de idade e o subteste Figura-Fundo na faixa etária dos 7 anos apresentam coeficientes abaixo de 0,80.

A consistência interna obtida a partir do coeficiente de Kuder-Richardson foi verificada de acordo com as faixas etárias específicas, assim como na amostra total, para cada subteste. Os dados encontram-se sumariados na Tabela 5. No subteste Analogias, foram excluídos 6 itens e, no subteste Dobra de Papel, foram excluídos 5 itens das análises pois os mesmos não apresentaram variância, ou seja, 100\% das crianças na amostra pontuaram zero.

De acordo com os dados apresentados na Tabela 5, os subtestes Formas Completas, Sequências e Padrões Repetidos apresentam boa consistência interna tanto em cada faixa etária quanto para a amostra total. Já o subteste FiguraFundo apresentou valores um pouco abaixo do esperado para todas as idades. O subteste Analogias apresentou melhor consistência interna para a faixa etária dos 7 anos em relação às outras idades. Por fim, o subteste Dobra de Papel apresentou baixa consistência interna, indicando problemas com a sua precisão.

Considerando as faixas etárias, a ANOVA revelou diferenças estatisticamente significativas nos subtestes entre os grupos. Os dados descritivos de média e desvio-padrão, bem como aqueles oriundos da ANOVA estão apresentados na Tabela 6

Tabela 4. Coeficientes de Spearman-Brown e correlações entre as duas metades dos subtestes de acordo com a faixa etária e o total

\begin{tabular}{ccccc}
\hline Subtestes & $\mathbf{6}$ anos & $\mathbf{7}$ anos & $\mathbf{8}$ anos & Total \\
\hline Figura-Fundo & $0,84(0,73)$ & $0,79(0,66)$ & $0,86(0,76)$ & $0,83(0,71)$ \\
Analogias & $0,72(0,56)$ & $0,86(0,76)$ & $0,81(0,69)$ & $0,81(0,69)$ \\
Formas Completas & $0,90(0,82)$ & $0,86(0,75)$ & $0,80(0,67)$ & $0,89(0,81)$ \\
Sequências & $0,96(0,92)$ & $0,97(0,94)$ & $0,97(0,95)$ & $0,97(0,95)$ \\
Padrões Repetidos & $0,94(0,88)$ & $0,94(0,88)$ & $0,92(0,86)$ & $0,94(0,89)$ \\
Dobra de Papel & $0,81(0,69)$ & $0,82(0,70)$ & $0,89(0,80)$ & $0,85(0,74)$ \\
\hline
\end{tabular}


Tabela 5. Valores de Kuder-Richardson por subteste de acordo com cada faixa etária e amostra total

\begin{tabular}{ccccccc}
\hline Subtestes & $\begin{array}{c}\text { Número } \\
\text { de itens }\end{array}$ & $\begin{array}{c}\text { Itens } \\
\text { analisados }\end{array}$ & $\mathbf{6}$ anos & $\mathbf{7}$ anos & $\mathbf{8}$ anos & Total \\
\hline Figura-Fundo & 31 & 31 & 0,77 & 0,73 & 0,73 & 0,77 \\
Analogias & 38 & 32 & 0,66 & 0,83 & 0,76 & 0,78 \\
Formas Completas & 36 & 36 & 0,86 & 0,81 & 0,80 & 0,86 \\
Sequências & 47 & 47 & 0,91 & 0,93 & 0,93 & 0,94 \\
Padrões Repetidos & 27 & 27 & 0,86 & 0,86 & 0,81 & 0,87 \\
Dobra de Papel & 26 & 21 & 0,72 & 0,51 & 0,65 & 0,65 \\
\hline
\end{tabular}

Tabela 6. Comparação nos subtestes e total da Leiter-R entre as faixas etárias

\begin{tabular}{ccccc}
\hline Subtestes/Idade & $\mathbf{6}$ anos & $\mathbf{7}$ anos & $\mathbf{8}$ anos & $\boldsymbol{F}$ \\
\hline Figura-fundo & $16,11( \pm 3,44)$ & $18,73( \pm 3,31)$ & $18,53( \pm 3,38)$ & $14,272^{*}$ \\
Analogias & $4,73( \pm 2,44)$ & $6,61( \pm 4,79)$ & $6,71( \pm 3,25)$ & $7,559^{*}$ \\
Formas Completas & $22,19( \pm 5,01)$ & $27,27( \pm 4,19)$ & $27,42( \pm 4,13)$ & $33,191^{*}$ \\
Sequências & $12,07( \pm 6,43)$ & $19,83( \pm 8,65)$ & $19,87( \pm 8,59)$ & $25,271^{*}$ \\
Padrões Repetidos & $13,09( \pm 5,11)$ & $17,18( \pm 4,50)$ & $16,61( \pm 3,75)$ & $18,069^{*}$ \\
Dobra de Papel & $4,57( \pm 1,92)$ & $5,73( \pm 1,73)$ & $6,34( \pm 1,90)$ & $17,129^{*}$ \\
Total & $72,71( \pm 17,61)$ & $95,18( \pm 19,21)$ & $95,48( \pm 18,45)$ & $38,741^{*}$ \\
\hline
\end{tabular}

$*_{p} \leq 0,01$

Tabela 7. Correlações entre a Leiter-R e os testes de inteligência WISC-III e Matrizes Progressivas Coloridas de Raven

\begin{tabular}{|c|c|c|c|c|c|c|c|}
\hline WISC-III/ Leiter & $\begin{array}{l}\text { Figura } \\
\text { Fundo }\end{array}$ & Analogias & $\begin{array}{c}\text { Formas } \\
\text { Completas }\end{array}$ & Sequências & $\begin{array}{c}\text { Padrões } \\
\text { Repetidos }\end{array}$ & $\begin{array}{c}\text { Dobra de } \\
\text { Papel }\end{array}$ & Total \\
\hline Total-Bruto & 0,34 & $0,37^{*}$ & 0,29 & $0,41^{*}$ & 0,17 & $0,37^{*}$ & $0,48 * *$ \\
\hline Verbal-Bruto & 0,31 & $0,39 *$ & $0,41^{*}$ & 0,26 & 0,17 & 0,25 & $0,43 * *$ \\
\hline Execução-Bruto & 0,29 & 0,22 & 0,19 & $0,46^{* *}$ & 0,20 & 0,31 & $0,44^{*}$ \\
\hline QI Total & $0,36^{*}$ & $0,39 *$ & 0,33 & $0,44^{*}$ & 0,21 & 0,31 & $0,51 * *$ \\
\hline QI Verbal & $0,37 *$ & $0,36^{*}$ & $0,43 *$ & 0,33 & 0,27 & 0,24 & $0,50 * *$ \\
\hline QI Execução & 0,23 & 0,27 & 0,12 & $0,41^{*}$ & 0,09 & 0,26 & 0,35 \\
\hline Informação & 0,32 & $0,38^{*}$ & 0,2 & $-0,04$ & 0,00 & 0,13 & 0,17 \\
\hline Semelhanças & 0,21 & 0,23 & 0,27 & 0,29 & 0,05 & $0,39 *$ & 0,34 \\
\hline Aritmética & 0,30 & 0,21 & $0,36^{*}$ & 0,05 & 0,12 & 0,17 & 0,28 \\
\hline Vocabulário & $-0,06$ & 0,00 & 0,17 & 0,18 & 0,16 & $-0,14$ & 0,13 \\
\hline Compreensão & 0,26 & $0,40^{*}$ & 0,28 & 0,25 & 0,17 & 0,24 & $0,37 *$ \\
\hline Dígitos & 0,27 & 0,19 & 0,34 & 0,14 & 0,19 & 0,06 & 0,3 \\
\hline Completar Fig. & $0,44^{*}$ & 0,14 & $0,44^{*}$ & $0,50 * *$ & 0,29 & 0,23 & $0,56^{* *}$ \\
\hline Códigos & $-0,13$ & $-0,12$ & $-0,21$ & 0,10 & 0,12 & $-0,05$ & $-0,03$ \\
\hline ArranjoFiguras & 0,23 & 0,14 & 0,33 & $0,35^{*}$ & 0,09 & 0,23 & $0,37 *$ \\
\hline Cubos & 0,34 & $0,47 * *$ & 0,22 & $0,51^{* *}$ & 0,13 & 0,35 & $0,49 * *$ \\
\hline ArmarObjetos & 0,32 & 0,12 & 0,17 & 0,14 & 0,09 & 0,33 & 0,26 \\
\hline Série A & $0,34 * *$ & $0,30^{*}$ & $0,25^{*}$ & $0,32 * *$ & $0,30 * *$ & $0,23^{*}$ & $0,42 * *$ \\
\hline SérieAb & $0,35^{* *}$ & 0,22 & $0,54 * *$ & $0,38^{* *}$ & $0,42 * *$ & $0,43 * *$ & $0,57^{* *}$ \\
\hline Série B & $0,40 * *$ & $0,34 * *$ & $0,55^{* *}$ & $0,48 * *$ & $0,50 * *$ & $0,35^{* *}$ & $0,65^{* *}$ \\
\hline Raven Total & $0,46 * *$ & $0,35^{* *}$ & $0,58 * *$ & $0,50 * *$ & $0,51 * *$ & $0,44 * *$ & $0,69 * *$ \\
\hline
\end{tabular}

*Correlação significativa ao nível de $p \leq 0,05$

** Correlação significativa ao nível de $p \leq 0,01$ 
Análises Post Hoc com correção de Bonferroni mostraram que as crianças com 6 anos de idade apresentam menores escores em relação às crianças de 7 e 8 anos, com diferenças estatisticamente significativas $(p<0,010)$ em todos os subtestes da Leiter-R, bem como nos escores totais. Entre as crianças de 7 e 8 anos de idade, não foram observadas diferenças significativas entre os subtestes e os escores totais, indicando desempenho semelhante em ambas as faixas etárias.

Análises para verificação de evidências de validade convergente foram realizadas a partir de correlações parciais, controlando o efeito da variável idade, entre os escores obtidos na Leiter-R com a WISC-III e com as Matrizes Progressivas Coloridas de Raven. Nas correlações com a WISC-III, foram utilizados os escores brutos e padronizados para QI Total, QI de Execução e QI Verbal, bem como os escores brutos dos subtestes. Para as análises de correlação com as Matrizes Progressivas Coloridas de Raven, foram considerados os totais brutos nas séries $\mathrm{A}, \mathrm{Ab}, \mathrm{B}$ e pontuação bruta Total. Os dados são apresentados na Tabela 7.

De acordo com os dados apresentados na Tabela 7, foram observadas correlações positivas entre os escores da Leiter-R e as medidas gerais da WISC-III. De modo geral, as correlações apresentaram magnitudes baixas a moderadas. No entanto, apenas alguns subtestes da Leiter-R apresentaram significância estatística na correlação com a WISC-III. Em relação aos escores totais, foram verificadas correlações significativas e de magnitude moderada entre o Total na Leiter-R e a pontuação bruta Total, Verbal e de Execução da WISC-III, assim como com o QI Verbal e o QI Total. Os subtestes da Leiter-R que apresentaram maior número de correlações significativas com a WISC-III foram Analogias e Sequências.

O mesmo procedimento foi realizado com os subtestes específicos da WISC-III. Os dados apresentados na Tabela 7 mostram que, de modo geral, a maioria das correlações foi positiva, com algumas exceções de correlações negativas entre os subtestes Códigos, Vocabulário e Informação da WISCIII com alguns subtestes da Leiter-R. Foram observadas correlações de magnitude baixa a moderada, embora apenas algumas tenham apresentado significância estatística, como aquelas observadas entre os subtestes Figura-Fundo e Completar Figuras; Analogias e Informação; Cubos e Compreensão; Formas Completas com Completar Figuras e Aritmética; Sequências com Completar Figuras, Arranjo de Figuras e Cubos; Dobra de Papel com Semelhanças; assim como o Total na Leiter-R se correlacionou com Completar Figuras, Arranjo de Figuras, Cubos e Compreensão. Não foram observadas correlações significativas entre o subteste Padrões Repetidos da Leiter-R com os subtestes da WISC-III.

Assim como em relação à WISC-III, foram realizadas correlações parciais, controlando o efeito da idade, entre o desempenho na Leiter-R e nas Matrizes Progressivas Coloridas de Raven. De acordo com os resultados obtidos, observa-se que todas as correlações foram positivas e significativas, com exceção do subteste Analogias e a Série $\mathrm{Ab}$ do Raven, embora haja uma tendência entre ambas as medidas $(p=0,060)$. A magnitude variou entre baixa $\mathrm{e}$ moderada, sendo que, de modo geral, as correlações tenderam a aumentar da série A para a série B, sendo maiores entre as medidas da Leiter-R e o Total no Raven.

\section{Discussão}

O presente artigo objetivou investigar evidências de validade e fidedignidade da BVR da Leiter-R em crianças pertencentes à faixa etária dos 6 aos 8 anos. Primeiramente foram verificadas evidências de validade baseadas na estrutura interna do instrumento. As correlações obtidas entre os subtestes da Leiter-R apresentaram maiores magnitudes em relação ao observado por Roid e Miller (1997). Entretanto, esses autores avaliaram crianças dos 6 aos 10 anos, enquanto que o presente estudo limitou-se à faixa etária dos 6 aos 8 anos de idade.

Os achados oriundos das AFE mostraram diferenças em relação aos modelos esperados, tanto o teórico quanto o observado na amostra americana (Roid \& Miller, 1997). Os subtestes se agruparam de formas diferentes, com alterações nos valores das cargas fatoriais. Também foram verificadas algumas diferenças em relação a um estudo prévio, realizado por Mecca (2010) com crianças na faixa etária dos 6 anos de idade. Neste, Figura-Fundo, Analogias e Sequências se agrupam em um primeiro fator enquanto que Formas Completas, Padrões Repetidos e Dobra de Papel se agrupam em um segundo fator. As diferenças para os achados do presente estudo encontram-se na mudança de agrupamento dos subtestes Figura-Fundo e Sequências. Os resultados do estudo de Mecca (2010) são mais semelhantes aos achados do estudo americano (Roid \& Miller, 1997), embora a amostra tenha sido relativamente menor do que o presente estudo e com uma faixa etária mais restrita. As diferenças observadas podem ocorrer em função dos diferentes métodos de extração dos fatores, uma vez que, no manual americano, não são apresentados detalhadamente os procedimentos realizados. Além disso, no presente estudo, foi utilizada análise paralela, considerada mais adequada do que critérios mais tradicionais utilizados no estudo de Mecca (2010).

A fidedignidade foi avaliada a partir da estabilidade teste e re-teste e pelos coeficientes de Kuder-Richardson e Spearman-Brown na amostra total e por faixa etária. Os dados obtidos a partir da Correlação de Spearman entre as versões teste e re-teste indicaram correlações positivas, a maioria de magnitude moderada entre as aplicações, assim como observado no estudo americano para os subtestes da Leiter-R (Roid \& Miller, 1997). Apenas para a medida da pontuação total é que foi observada correlação de alta magnitude, assim como observado por Roid e Miller (1997). No entanto, comparando os resultados de ambas as amostras, verifica-se que, no geral, os valores obtidos a partir da amostra americana são maiores em relação ao observado no presente estudo. As maiores discrepâncias foram observadas nos subtestes Formas Completas e Sequências, com valores de Kuder-Richardson expressivamente maiores na amostra americana, enquanto que, nos subtestes Padrões Repetidos e Figura-Fundo, as diferenças foram menores.

No estudo de Roid e Miller (1997), foi realizada Correlação de Pearson, enquanto que, no presente estudo, optou-se pela Correlação de Spearman em função do 
número reduzido de sujeitos que realizaram o re-teste. Valores inferiores encontrados no presente estudo podem ser justificados pela diferença no número de sujeitos avaliados, uma vez que no estudo americano a amostra foi composta por 48 crianças. Além do mais, a amostra americana foi composta por crianças de até 10 anos de idade, enquanto que, neste estudo, foram incluídas crianças somente até os 8 anos. Vale ressaltar que foram utilizadas pontuações diferentes uma vez que no presente estudo foram considerados os totais brutos enquanto que, no estudo de Roid e Miller, foram consideradas as pontuações ponderadas e o QI Total.

Em relação aos resultados obtidos a partir do Método das Metades, observam-se valores adequados, que refletem homogeneidade dos subtestes, principalmente na faixa etária dos 8 anos e na amostra total. No grupo de crianças com 6 anos de idade, os valores do Coeficiente de Spearman-Brown foram adequados em todos os subtestes, exceto no subteste Analogias. Enquanto que, aos 7 anos, o subteste FiguraFundo não apresentou valor dentro do esperado. No entanto, embora abaixo de 0,80 , esses dois valores ainda são aceitáveis desde que a precisão do instrumento não seja limitada a apenas esses dados (Conselho Federal de Psicologia [CFP], 2003; Urbina, 2014). Ao analisar os dados de cada subteste em função das faixas etárias, observa-se que, em Analogias e Dobra de Papel, há um aumento da precisão dos 6 para 7 e 8 anos de idade. Enquanto que, no subteste Formas Completas, há uma tendência à diminuição da precisão conforme aumento das idades. Já os subtestes Sequências e Padrões apresentam, de modo geral, uma precisão estável entre os grupos etários. Por fim, no subteste Figura-Fundo, são observados valores maiores nos grupos de 6 e 8 anos em relação ao grupo de 7 anos de idade.

Considerando os dados obtidos a partir da amostra total, os subtestes Sequências e Padrões Repetidos apresentam maiores coeficientes quando comparados aos subtestes Figura-Fundo, Analogias, Formas Completas e Dobra de Papel, embora estes apresentem valores adequados. Esses dados replicam os achados de um estudo prévio realizado por Mecca (2010) com crianças em fase pré-escolar, indicando que a variação dos coeficientes em diferentes grupos etários se assemelha nos subtestes da Leiter-R.

Como indicativo da adequação da consistência interna dos subtestes da Leiter-R foi analisado o coeficiente alfa. Considerando como ponto de corte o valor de 0,70 , o que representa cerca de $50 \%$ de ausência de erro, observa-se que, ao longo das faixas etárias, assim como na amostra total, os subtestes Figura-Fundo, Formas Completas, Sequências e Padrões Repetidos apresentam índices satisfatórios, com exceção do subteste Dobra de Papel para crianças de 7 e 8 anos, além do subteste Analogias para crianças aos 6 anos de idade. Esses resultados se assemelham aos achados de Mecca (2010), em que os coeficientes alfa na amostra total são satisfatórios na maioria dos subtestes, exceto para Analogias e Dobra de Papel. Os resultados de ambos os estudos também se assemelham em relação às crianças na faixa etária dos 6 anos de idade, em que maiores valores são observados nos subtestes Formas Completas, Sequencias e Padrões Repetidos, enquanto que índices não satisfatórios são observados no subteste Analogias. Entretanto, no presente estudo, observa-se adequação do subteste Dobra de Papel a essa faixa etária, o que não ocorreu no estudo de Mecca. Essa diferença pode estar associada ao maior número de sujeitos que participaram do presente estudo.

Comparando os índices de consistência interna com os achados do estudo de normatização americano (Roid \& Miller, 1997), observam-se valores muito semelhantes ao longo das faixas etárias em ambas as amostras nos subtestes Figura-Fundo e Formas Completas. No entanto, para os subtestes Sequências e Padrões Repetidos, os índices do presente estudo são mais altos que os de Roid e Miller (1997). No subteste Analogias, os índices se assemelham aos 7 anos de idade, embora, no presente estudo, tenham sido menores aos 6 e 8 anos quando comparados aos dados de Roid e Miller. Inversamente, no subteste Dobra de Papel, os índices são similares aos 6 e 8 anos, enquanto que, no presente estudo, são menores aos 7 anos de idade. Porém, os resultados de ambos os estudos demonstram valores insatisfatórios do coeficiente alfa do subteste Dobra de Papel para crianças de 7 e 8 anos de idade, demonstrando ser um problema do instrumento em si e não das diferentes amostras.

Em relação às evidências de validade, as análises de variância mostraram que há uma tendência ao longo do desenvolvimento para aumento dos escores dos 6 para os 7 e 8 anos de idade em tarefas que requerem habilidades de exploração, discriminação e síntese visual. O mesmo foi observado em habilidades mais complexas de raciocínio indutivo e sequencial. Diferenças entre faixas etárias costumam ser vistas como evidências de validade dos testes, pois, na maioria deles, o desempenho de crianças e adolescentes das amostras normativas mostra tipicamente uma tendência ascendente em idades cronológicas sucessivas (Urbina, 2007).

No entanto, é comum crianças de diferentes idades a partir dos 6 anos, agruparem-se em uma mesma faixa etária no processo de normatização de testes (Angelini et al., 1999; Figueiredo, 2002; Sisto, Noronha, \& Santos, 2006) uma vez que não apresentam diferenças significativas em seus escores. Apesar de consistente com a literatura, esses achados diferem dos dados americanos, uma vez que as normas foram elaboradas dividindo-se a amostra em grupos etários de 3 em 3 meses. Vale ressaltar que, no estudo de Roid e Miller (1997), não há dados referentes à significância da diferença entre os escores dos diferentes grupos e há um número reduzido de sujeitos por grupo. Nesse sentido, podemos citar o grupo de 101 crianças com 6 anos de idade que foi dividido em 4 grupos diferentes: dos 6 anos a 6 anos e 2 meses; 6 anos e 3 meses a 6 anos e 5 meses; 6 anos e 6 meses a 6 anos e 8 meses; 6 anos e 9 meses a 6 anos e 11 meses (Roid \& Miller, 1997).

Os achados do presente estudo que se referem à diferença entre crianças de 6 para 7 e 8 anos de idade replicam dados prévios da literatura. O estudo de Bandeira, Alves, Giacomel e Lorenzatto (2004) apontou que crianças na faixa etária dos 7 anos de idade não se diferenciam de crianças cuja idade varia de 8 anos a 8 anos e 5 meses. Crianças na faixa etária dos 6 anos, por sua vez, apresentam diferenças estatisticamente significativas quando comparadas a crianças com 8 anos em tarefas de raciocínio não-verbal que requerem dedução de relações, tais como os subtestes da Leiter-R Analogias, Sequencias e Padrões Repetidos. 
Evidências de validade convergente foram verificadas a partir das correlações de magnitude moderada entre o escore total na Leiter-R com os escores brutos da WISC-III em sua pontuação total, verbal e de execução. $\mathrm{O}$ mesmo foi observado entre a Leiter-R e os índices de QI Total e QI Verbal da WISC-III. Esses achados se assemelham ao encontrado por Cathers-Schiffmane e Thompson (2007) no que tange às medidas globais da WISC-III e da Leiter-R. Por se tratar de instrumentos desenvolvidos a partir de modelos teóricos distintos, de fato, uma relação moderada era esperada, de modo que apenas uma parte da variância seja explicada por um fator comum. Além disso, a Leiter-R não contém itens verbais ou que demandam velocidade de processamento tais como observados na WISC-III.

Em relação aos estudos da amostra normativa realizados por Roid e Miller (1997), estes encontraram correlações mais altas entre as medidas da WISC-III e da Leiter-R quando comparadas aos resultados do presente estudo. Porém, tanto o estudo de Cathers-Schiffmane e Thompson (2007) como o de Roid e Miller utilizaram os escores padronizados da Leiter-R, sendo que, no presente estudo, foram utilizados os dados brutos, uma vez que o instrumento ainda se encontra em processo de estudo sobre suas propriedades psicométricas.

Entre os subtestes específicos, observa-se que Analogias, Sequências e Dobra de Papel apresentam correlações estatisticamente significativas com o Total na WISC-III. Isso indica que habilidades de raciocínio analógico e sequencial, bem como habilidades de manipulação mental de imagens avaliadas pela Leiter-R, estão relacionadas ao desempenho geral na WISC-III. Analogias e Formas Completas, por sua vez, apresentam correlações com o escore Total Verbal. Nesse sentido, habilidades de raciocínio analógico e síntese visual, embora não requeiram a manipulação de estímulos verbais, estão associadas ao desempenho em tarefas que, de modo geral, demandam respostas e compreensão de instruções verbais. Por fim, somente o subteste Sequências se correlacionou com o escore Total de Execução, indicando uma relação entre habilidades não-verbais visuo-espaciais de atenção seletiva a estímulos visuais e visuo-construtivas da WISC-III com raciocínio sequencial, uma habilidade específica da inteligência fluida.

O subteste Figura-Fundo da Leiter-R se correlacionou com o subteste Completar Figuras da WISC-III. Ambos são os primeiros subtestes a serem aplicados em cada bateria e avaliam habilidades de exploração e discriminação visual. No primeiro é solicitado ao sujeito que encontre em uma prancha com estímulos distratores ao estímulo-alvo apresentado (Roid \& Miller, 1997; Mecca, 2010). Enquanto que no segundo solicita-se apontar para a parte que está faltando no desenho (Figueiredo, 2002). Desta forma, ambos exigem o rastreio visual.

O subteste Completar Figuras da WISC-III também se correlacionou moderadamente com o subteste Formas Completas da Leiter-R, sendo este uma medida de exploração, manipulação e síntese visual. Formas Completas se correlacionou com Aritmética, indicando que habilidades de manipulação visual podem estar associadas a tarefas que requerem manipulação mental de informações para resolução de problemas aritméticos. Isso corrobora achados prévios sobre a relação entre processamento visual e aritmética (Geary, 2007; Hale et al., 2008).

O subteste Analogias se correlacionou com Cubos, ou seja, com o desempenho em uma tarefa que requer habilidades espaciais e visuo-construtivas. Também se correlacionou com dois subtestes verbais, Informação e Compreensão, que avaliam conhecimentos adquiridos em ambientes formais de aprendizagem e compreensão de situações e contextos, respectivamente. O raciocínio sequencial avaliado pelo subteste Sequencias se correlacionou com Completar Figuras, Cubos e Arranjo de Figuras, sendo que, nesta última tarefa, o objetivo é ordenar estímulos em uma sequência correta de modo que forme uma história coerente. Por fim, o subteste Dobra de Papel se correlacionou com Semelhanças, indicando relação entre uma tarefa que avalia manipulação mental de imagens e outra que avalia memória semântica.

Comparando com os achados entre a Leiter-R e a WISCIII, correlações mais elevadas foram observadas entre os escores na Leiter-R e nas Matrizes Progressivas Coloridas de Raven. Considerando as características dos itens de cada teste, maiores correlações da Leiter-R com o Raven eram esperadas em relação à WISC-III, pois ambos os instrumentos são compostos somente de estímulos visuais. Apesar de executarem tarefas com objetivos distintos, para responder tanto ao Raven como à Leiter-R, os indivíduos precisam manipular mentalmente estímulos visuais, ter um raciocínio visuo-espacial e estabelecer relações entre estímulos (raciocínio indutivo). Apesar das correlações com o Raven terem sido maiores, a magnitude foi moderada, indicando que há, em ambos os instrumentos, aspectos distintos e específicos apesar de compartilharem parcialmente uma variância comum.

As medidas da Leiter- $\mathrm{R}$ se correlacionaram com a pontuação total no Raven e com as séries $\mathrm{A}, \mathrm{Ab}$ e B, que avaliam a capacidade para completar um padrão contínuo que pode mudar em uma ou duas direções de acordo com o nível de dificuldade, capacidade de perceber figuras discretas como um todo e habilidades de apreensão de mudanças análogas em figuras relacionadas espacialmente e logicamente, respectivamente (Angelini et al., 1999). A exceção se deu entre o subteste Analogias e a Série Ab, entre as quais não foram observadas correlações.

\section{Considerações Finais}

Os resultados obtidos a partir deste estudo indicam adequação dos parâmetros psicométricos da Leiter-R que foram investigados para crianças entre 6 e 8 anos de idade. A estrutura interna, apesar de algumas divergências em relação ao esperado, apresentou bons índices de ajuste, mostrando-se adequada na interpretação de dois fatores predominantes.

Os escores na Leiter-R mostraram-se estáveis ao longo do tempo, bem como apresentou adequação da homogeneidade dos itens em função das diferentes faixas etárias. O mesmo foi observado em relação à consistência interna, uma vez que somente o subteste Dobra de Papel apresentou problemas nos itens, principalmente nos grupos de 7 e 8 anos.

Tendências ao longo do desenvolvimento foram verificadas a partir do aumento do desempenho com a 
idade. Para estudos futuros, sugere-se aumento da amostra para divisão dos grupos em seis meses. Evidências de validade convergente foram verificadas a partir das correlações observadas entre a Leiter-R e outras medidas reconhecidamente utilizadas na avaliação de inteligência. Maiores correlações foram observadas com as Matrizes Progressivas Coloridas de Raven, indicando que, de fato, a Leiter-R se relaciona mais com tarefas que envolvem habilidades visuo-espaciais e de raciocínio do que com testes que requerem uma demanda verbal. Neste estudo, foram realizadas correlações com o agrupamento dos itens de acordo com as séries do Raven que estão apresentadas no manual. Outra possibilidade seria agrupar os itens por fatores, conforme verificado por Simões (2000), que avaliam habilidades distintas como raciocínio concreto e abstrato por analogia; completar um padrão simples e descontínuo; e completar padrões através de estruturação e raciocínio concreto por analogia. Nesse sentido, sugerem-se análises futuras que correlacionem as médias da Leiter-R com esses componentes.

As análises realizadas pautam-se na Teoria Clássica dos Testes (TCT), que utiliza os escores totais nas análises, sugerindo-se futuros estudos baseados na Teoria de Resposta ao Item (TRI), que possam verificar parâmetros de adequação dos itens que compõem a Leiter-R. Os resultados do presente estudo podem contribuir para pesquisas que objetivam avaliar crianças com distúrbios do desenvolvimento. A Leiter-R é um instrumento de avaliação que permite identificar um perfil cognitivo de diferentes condições clínicas. Dessa forma, este estudo apresenta dados preliminares de uma amostra de crianças dos 6 aos 8 anos de idade que podem ser utilizados como referência em estudos futuros de comparação de grupos.

\section{Referências}

American Educational Research Association, American Psychological Association, \& National Council on Measurement in Education. (2014). Standards for Educational and Psychological Testing. Washington: American Educational Research Association.

Anastasi, A., \& Urbina, S. (2000). Testagem Psicológica. Porto Alegre: Artmed.

Anastasi, A. (1977). Testes Psicológicos. São Paulo: EPU.

Angelini, A. L., Alves, I. C.B., Custódio, E. M., Duarte, W. F., \& Duarte, J. L. (1999). Matrizes Progressivas Coloridas. São Paulo: CETEPP.

Antonio, D. A. M., Mecca, T. P., \& Macedo, E. C. (2012). O uso do teste não-verbal Leiter-R na avaliação de inteligência em distúrbios do desenvolvimento. Cadernos de Pós-graduação em Distúrbios do Desenvolvimento, 12(2), 9-15.
Bandeira, D. R., Alves, I. C. B., Giacomel, A. E., \& Lorenzatto, L. (2004). Matrizes Progressivas Coloridas de Raven - Escala Especial: Normas para Porto Alegre. Psicologia em Estudo, 9(3), 479-486.

Carroll, J. B. (1993). Human Cognitive Abilities: A Survey of Factor Analytic Studies. New York: Cambridge University Press.

Cathers-Schiffman, T. A., \& Thompson, M. L. (2007). Assessment of English- and Spanish-speaking Students with the WISCIII and Leiter-R. Journal of Psychoeducational Assessment, 25(1), 41-52.

Conselho Federal de Psicologia. (2003). Resolução 002 de 24 de março de 2003. Disponível em: http://www.pol.org.br/pol/cms/ pol/legislacao/resolucao/resolucao_2003_002.html.

Figueiredo, V. L. (2002). Escala Wechsler de Inteligência para Crianças ( $3^{\mathrm{a}}$ ed.). São Paulo: Casa do Psicólogo.

Geary, D. C. (2007). An evolutionary perspective on learning disability in mathematics. Developmental Neuropsychology, 32(1), 471-519.

Hale, J. B., Fiorello, C. A., Dumont, R., Willis, J. O., Rackley, C., \& Elliott, C. (2008). Differential Ability Scales-Second Edition (neuro) psychological predictors of math performance for typical children and children with math disabilities. Psychology in the Schools, 45(9), 838-858.

Hooper, V. S., \& Bell, S. M. (2006). Concurrent validity of the Universal Nonverbal Intelligence Test and Leiter International Performance Scale-Revised. Psychology in the Schools, 43(2), 143-148.

Mecca, T. P. (2010). Tradução, adaptação, fidedignidade e evidências de validade da Bateria de Visualização e Raciocinio da Leiter International Performance Scale Revised (Unpublished master's thesis). Programa de Pós-graduação em Distúrbios do Desenvolvimento, Universidade Presbiteriana Mackenzie, São Paulo.

Mecca, T. P., Antonio, D. A. M., Seabra, A. G., \& Macedo, E. C. D. (2014). Parâmetros psicométricos da Escala Internacional de Inteligência Leiter-R para Crianças Pré-Escolares. Avaliação Psicológica, 13(1), 125-132.

Roid, G. H., \& Miller, L. J. (1997). Leiter International Performance Scale-Revised. Wood Dale, IL: Stoelting.

Simões, M. M. R. (2000). Investigações no âmbito da Aferição Nacional do Teste das Matrizes Progressivas Coloridas de Raven (M. P. C.R). Lisboa: fundação Calouste Gulbenkian / Fundação para a Ciência e a Tecnologia.

Sisto, F. F., Noronha, A. P., \& Santos, A. A. A. (2006). TONI-3: Forma A. São Paulo: Vetor Editora.

Urbina, S. (2007). Fundamentos da testagem psicológica. Porto Alegre: Artmed.

Urbina, S. (2014). Essentials of psychological testing (2 ${ }^{\text {a }}$ ed.). John New York: Wiley \& Sons.

Watkins, M. W. (2006). Determining parallel analysis criteria. Journal of Modern Applied Statistical Methods, 5(2), 344-346. 